\title{
Factores de riesgo y letalidad asociados a Candidemia Neonatal en una unidad de neonatología
}

\author{
Risk factors and lethality associated with Neonatal Candidemia in a neonatal unit
}

\section{Elizabeth Caparó Ingram ${ }^{a}$, Manuel Vásquez Vega ${ }^{a}$, Ximena Norero $^{a}$, Xavier Sáez-Llorens ${ }^{\mathrm{a}}$, Rodrigo DeAntonio ${ }^{\mathrm{b}}$, Edgar Rodríguez Barría ${ }^{\mathrm{c}}$}

\author{
aHospital del Niño Dr. José Renán Esquivel, Panamá \\ bInstituto Nacional de Salud Pública, México \\ 'Hospital Dr. Manuel Nieto, Darién, Panamá
}

Recibido: 9 de mayo de 2018; Aceptado: 27 de noviembre de 2018

\section{Resumen}

Objetivo: Determinar los principales factores de riesgo asociados a candidemia en neonatos. Pacientes y Método: Se realizó un estudio de casos y controles retrospectivo pareado, desde enero de 2014 a diciembre de 2016. Los casos fueron pacientes con aislamiento en hemocultivo y/o líquido cefalorraquídeo de Candida spp. luego de sus primeras 48 horas intrahospitalarias y los controles fueron neonatos escogidos del censo estadístico de la sala de neonatología pareados según su fecha de admisión a sala (rango de 30 días), peso al nacer, edad gestacional y condición de egreso (vivo o fallecido). Por cada caso seleccionamos dos controles. Los factores de riesgo evaluados fueron la estancia intrahospitalaria mayor a 7 días, el uso de antibióticos de amplio espectro, ventilación mecánica, nutrición parenteral mayor a 5 días, procedimientos invasivos como colocación de accesos venosos centrales y cirugías abdominales y torácicas, enterocolitis necrosante y crecimiento de microorganismo bacteriano en hemocultivo previo a la candidemia. Resultados: Durante el período de estudio hubo aislamiento de Candida spp. en 141 neonatos. Se encontró que el 49\% fue de Candida parapsilosis con la mayor letalidad asociada. En el análisis multivariado se identificaron como factores de riesgo la estancia intrahospitalaria mayor a 7 días $(\mathrm{OR}=17,0,95 \% \mathrm{IC}=2,36-122,4)$, uso de líneas umbilicales $(\mathrm{OR}=9,04,95 \% \mathrm{IC}=1,55-52,5)$, exposición a cirugía $(\mathrm{OR}=12,4,95 \% \mathrm{IC}=1,76-87,3)$ y el uso de meropenem $(\mathrm{OR}=4,62,95 \% \mathrm{IC}=1,34-15,9)$. Conclusión: La estancia intrahospitalaria prolongada mayor a 7 días y la exposición a cirugía fueron los factores de riesgo más importantes en este estudio para el desarrollo de candidemia neonatal.
Palabras clave:

Neonato;

Candidemia;

Factores de riesgo;

Fluconazol;

Panamá 


\begin{abstract}
Objective: To identify the main risk factors associated with neonatal candidemia. Patients and Method: A retrospective paired case-control study was conducted from January 2014 to December 2016. The cases were patients with isolation in blood culture and/or cerebrospinal fluid of Candida spp. after their first 48 hours in the hospital and the controls cases were neonates chosen from the statistical census of neonatology paired according to their admission date (30-day range), birth weight, gestational age, and discharge condition (alive or deceased). For each case, we select two controls. The risk factors evaluated were intrahospital stay over seven days, use of broad-spectrum antibiotics, mechanical ventilation, parenteral nutrition longer than five days, invasive procedures such as central venous access and abdominal and thoracic surgeries, necrotizing enterocolitis and growth of bacterial microorganisms in blood culture before candidemia. Results: During the study period, 141 patients developed candidemia. 49\% of the cases corresponded to Candida parapsilosis with the highest associated lethality rate. The multivariate analysis identified as risk factors hospital stay longer than seven days $(\mathrm{OR}=17.0,95 \% \mathrm{CI}=2.36-122.4)$, use of umbilical lines $(\mathrm{OR}=9.04,95 \%$ $\mathrm{CI}=1.55-52.5)$, abdominal and/or thoracic surgery $(\mathrm{OR}=12.4,95 \% \mathrm{CI}=1.76-87.3)$, and treatment with Meropenem $(\mathrm{OR}=4.62,95 \% \mathrm{CI}=1.34-15.9)$. Conclusion: Prolonged intrahospital stay longer than seven days and thoracic and/or abdominal surgery were the most significant risk factors in this study for the development of neonatal candidemia.
\end{abstract}

Keywords:

Neonate;

Candidemia;

Risk factors;

Fluconazole;

Panama

\section{Introducción}

Actualmente las infecciones por Candida spp. se asocian a una mayor mortalidad en la población neonatal, por lo que es necesario evaluar los principales factores de riesgo asociados a este proceso infeccioso.

Los pacientes en las unidades de cuidados intensivos neonatales (UCIN) son colonizados por especies de Candida spp. poco tiempo después de nacer, encontrándose colonización principalmente en el tracto gastrointestinal y el respiratorio, en las dos primeras semanas de vida por transmisión vertical asociada al parto. Luego de dos semanas de vida el sitio de colonización más frecuente es la piel, esto puede estar relacionado a la manipulación del paciente por el personal de salud. ${ }^{1,2}$

Los factores de riesgo para candidemia en los pacientes prematuros están asociados a inmadurez del tracto gastrointestinal, virulencia de la cepa colonizadora, deficiencias e inmadurez inmunológica y al desarrollo incompleto de la epidermis que debilita las barreras físicas e inmunológicas facilitando la invasión. Luego de tener acceso a las mucosas o al torrente sanguíneo, Candida spp. muestra predilección por la invasión del sistema nervioso central, los riñones, el hígado, el bazo, el corazón y la retina, con las complicaciones correspondientes al compromiso de la función de dichos órganos. ${ }^{3,4}$

Candida es la principal causa de infecciones invasivas por hongos en la UCIN y es el tercer microorganismo más comúnmente aislado en hemocultivos en sepsis neonatal tardía. Se estima que es la causa del 2 al $4 \%$ de las infecciones en sepsis neonatal temprana y del 10 al $12 \%$ en sepsis neonatal tardía. ${ }^{5}$
La candidiasis neonatal incrementa la mortalidad y morbilidad de manera significativa, con una tasa de mortalidad entre 15 a $60 \%$, siendo más alta en los neonatos de extremo bajo peso al nacer EBPN (menor e igual a $1.000 \mathrm{~g})^{5}$.

El objetivo de este estudio fue determinar los principales factores de riesgo asociados a candidemia en neonatos atendidos en el Hospital del Niño Dr. José Renán Esquivel de Panamá (DRJRE).

\section{Pacientes y Método}

Se realizó un estudio de casos y controles retrospectivo pareado entre enero de 2014 y diciembre de 2016. Ante la alta prevalencia de candidemia en la unidad de neonatología de la institución, previamente conocida, se decidió tomar tres años para el periodo de estudio.

Los casos fueron todos los neonatos que tuvieron hemocultivo y/o líquido cefalorraquídeo con aislamiento de Candida spp. luego de $48 \mathrm{~h}$ de hospitalización en la sala de neonatología del Hospital del Niño DRJRE, ingresados en el periodo de estudio. Los controles fueron tomados del censo estadístico de la sala de neonatología pareados según su fecha de ingreso, peso al nacer, edad gestacional y condición de egreso. Para embarazos múltiples solo se seleccionó uno de los productos como control. Luego se evaluaron los expedientes clínicos en los archivos del Hospital del Niño DRJRE.

Se calculó la muestra utilizando openepi teniendo un nivel de confianza de 95 , porcentaje de probabilidad de detección de 80, con razón de controles por casos en 2, proporción hipotética de controles con exposición de $4 \%$, proporción hipotética de casos con exposición 
de $13 \%$ y odds ratios de 3.59 obteniendo según Kelsey un tamaño mínimo de casos de 76 y según Fleiss de 89 y un tamaño mínimo de muestra de controles según Kelsey de 152 y según Fleiss de 178 siendo un total de 267 pacientes.

\section{Población de referencia y lugar de estudio}

El estudio se realizó en la sala de neonatología del Hospital del Niño DRJRE, con capacidad para 90 pacientes ( 2 unidades de cuidados intensivos con 14 espacios cada una, 3 áreas de cuidados intermedios con 20 a 24 pacientes en cada una y un área de aislamiento con capacidad para 2 pacientes), a la que fueron admitidos 6.755 neonatos durante el periodo del estudio.

Se define EBPN recién nacido menor de $1.000 \mathrm{~g}$, muy bajo peso al nacer (MBPN) recién nacido menor de $1.500 \mathrm{~g}$ y bajo peso al nacer (BPN) al recién nacido menor de $2500 \mathrm{~g}^{6}$.

Durante el estudio todos los pacientes menores de $1.250 \mathrm{~g}$ recibieron profilaxis con fluconazol a una dosis intravenosa de $3 \mathrm{mg} / \mathrm{kg}$ cada $48 \mathrm{~h}$, iniciando la primera dosis al tercer día de vida y continuando la profilaxis por un período de seis semanas. Este protocolo se está aplicando en la institución desde el año $2009^{7}$.

\section{Método microbiológico}

Se inoculó los especímenes de sangre en las botellas pediátricas aeróbicas BacT/AlerT PF Plus (Biomérieux, Francia), luego fueron incubadas en el sistema automatizado BacT/AlerT 3D por un periodo de 5 días. El volumen de sangre extraído para el hemocultivo se determinó según el peso del paciente: $1 \mathrm{ml}$ para pesos menor o iguales a $1.000 \mathrm{~g}, 2 \mathrm{ml}$ para pesos de $1.001 \mathrm{a}$ $3.000 \mathrm{~g} \mathrm{y} 4 \mathrm{ml}$ para pesos mayores a $3.001 \mathrm{~g}$. Se les realizó tinción de gram a las botellas que fueron positivas, cada muestra con evidencia microscópica de levaduras fueron subcultivada en agar sabouraud e incubadas a $35+/-2$ o $\mathrm{C}$ por $24 \mathrm{~h}$. La identificación fenotípica y prueba de susceptibilidad para cada aislamiento se realizó usando las tarjetas YST y AST-YBC en el sistema Vitek2 (Biomérieux, Francia). Las muestra de líquido cefalorraquídeo se inocularon en caldo tioglicolato y se incubó a $37^{\circ} \mathrm{C}$ por $72 \mathrm{~h}$. Al crecer levaduras en el medio sólido se realizó el proceso de identificación y prueba de susceptibilidad de igual forma que el hemocultivo.

\section{Plan de análisis}

Inicialmente se realizó un análisis descriptivo de las características de los casos y controles. Se seleccionaron las variables propuestas como factores de riesgo en la literatura y se estableció la presencia o ausencia de estas asociaciones en la sala de neonatología. A través de la comparación entre los casos y los controles, se realizó un análisis univariado estimando el Odds Ratio (OR) como la medida en que estos factores de riesgo predisponen a los pacientes a desarrollar candidemia, definiendo como estadísticamente significativo un valor de $p<0.05$. Todas las variables con valor de $p<0.05$ fueron seleccionadas para la inclusión en el modelo de regresión logística multivariado. El análisis se realizó mediante el programa estadístico STATA 14.

La elaboración del estudio actual se rige por las guías éticas internacionales de estudios epidemiológicos elaboradas por la Organización Mundial de la

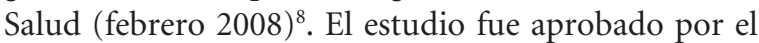
comité de bioética de la institución y por el comité de bioética del Ministerio de Salud. Se obtuvo la autorización escrita de ambos comités para efectuar la revisión de los expedientes clínicos.

\section{Resultados}

Se admitieron 6755 recién nacidos a la sala de neonatología desde enero de 2014 a diciembre de 2016 de los cuales, $2801(41.5 \%)$ fueron admitidos a UCIN y 3954 (58.5\%) al área de cuidados intermedios.

Se reportaron 836 hemocultivos positivos de los cuales 328 episodios (39.2\%) fueron microorganismos Gram negativos, 263 (31.5 \%) hongos y 245 (29.3\%) Gram positivos. De los 263 hemocultivos reportados con crecimiento por hongos se identificaron 141 episodios de candidemia en 141 pacientes, correspondiendo a un episodio de candidemia por paciente; de éstos se excluyeron 7 pacientes ya que presentaron crecimiento por Candida previo a las $48 \mathrm{~h}$ de hospitalización, teniendo un total de 134 pacientes con candidemia en el grupo de casos, de los cuales 3 pacientes (2\%) presentaron aislamiento de Candida en líquido cefalorraquídeo. El grupo control fue constituido por 268 pacientes.

La incidencia de candidemia fue de 20 por cada 1.000 admisiones, observándose la mayor tasa en los pacientes con EBPN (406.6 por cada 1.000 neonatos de EBPN). El incremento en la misma fue de 15 por 1.000 admisiones en el 2014 a 30 por 1.000 admisiones en el 2015 , teniendo un descenso para el año 2016 a 18 por 1.000 admisiones.

Al evaluar la persistencia de candidemia (definiéndose como hemocultivo por Candida seis días luego de haber iniciado terapia antifúngica $)^{5}$ versus la no persistencia se encontró que no hubo relación entre la persistencia y la mortalidad, que fue de $59 \%$ en la candidemia persistente versus un $63 \%$ en la no persistente.

\section{Características generales de los casos y controles}

Las características de sexo, edad gestacional, peso de nacimiento y estado al egreso se describen en la tabla 1, y fueron similares en los casos y los controles.

De 273 neonatos con EBPN y MBPN (<1.500 g), 91 $(33 \%)$ desarrollaron candidemia y de 68 neonatos con 
Tabla 1. Características clínicas de los casos y controles

\begin{tabular}{lccc}
\hline Variable & $\begin{array}{c}\text { Caso } \\
\mathrm{n}=134\end{array}$ & $\begin{array}{c}\text { Control } \\
\mathrm{n}=268\end{array}$ & Valor $\mathrm{p}$ \\
\hline $\begin{array}{l}\text { Demográficos } \\
\text { Sexo }\end{array}$ & & & \\
$\quad$ Masculino & $81(60,5 \%)$ & $165(61,5 \%)$ & 0,82 \\
$\quad$ Edad gestacional & & & \\
$\quad<28$ semanas & $42(31,3 \%)$ & $67(25,0 \%)$ & 0,17 \\
$\quad<32$ semanas & $80(59,7 \%)$ & $159(59,3 \%)$ & 0,94 \\
$\quad<36$ semanas & $106(79,1 \%)$ & $208(77,6 \%)$ & 0,73 \\
Peso nacer (g) & & & \\
$\quad<1.500 \mathrm{~g}$ & $91(67,9 \%)$ & $182(67,9 \%)$ & 1,00 \\
$\quad<2.500 \mathrm{~g}$ & $115(85,8 \%)$ & $226(84,3 \%)$ & 0,69 \\
Condición de egreso & & & \\
$\quad$ Vivo & $51(38,0 \%)$ & $103(38,0 \%)$ & - \\
$\quad$ Fallecido & $83(62,0 \%)$ & $165(62,0 \%)$ & - \\
\hline
\end{tabular}

Caso y Controles (razón de 1:2), fueron pareados según edad gestacional, peso al nacer, fecha de ingreso a la sala y condición de egreso.

BPN (<2.500 g), 24 (35\%) desarrollaron candidemia. Durante el estudio 191 (100\%) de los pacientes menores de $1.250 \mathrm{~g}$ recibieron profilaxis con fluconazol, desarrollándose candidemia en 80 pacientes $(42 \%)$.

Se encontró una tasa de letalidad global por candidemia de $62 \%$. La letalidad por especie se describe en la tabla 2.

Candida parapsilosis causó 66 (49\%) episodios de

\begin{tabular}{lc}
$\begin{array}{l}\text { Tabla 2. Tasa de letalidad de candidemia según } \\
\text { especie }\end{array}$ \\
\hline Especie & Letalidad (\%) \\
\hline Candida parapsilosis & $68 \%$ \\
Candida guilliermondii & $67 \%$ \\
Candida albicans & $55 \%$ \\
Candida tropicalis & $55 \%$ \\
Candida haemulonii & $50 \%$ \\
Candida lusitaniae & $50 \%$
\end{tabular}

Pacientes hospitalizados en la sala de neonatología de 2014 al 2016.

candidemia, en segundo lugar está Candida albicans con $44(33 \%)$ episodios, le siguen con menor porcentaje Candida tropicalis con 11 (8\%) episodios, Candida spp. con 7 (5\%) episodios, otras especies causaron 9 (7\%) episodios, Candida guilliermondii, Candida haemulonii y Candida lusitaniae causaron 3 (2\%), 2 $(1,4 \%), 2(1,4 \%)$ episodios respectivamente.

\section{Análisis univariado}

Inicialmente se evaluaron las variables de manera independiente, evidenciando los posible factores de riesgo para candidemia de manera individual (tabla 3 ). Al realizar el análisis univariado pareado, se observó

Tabla 3. Factores de Riesgo para Candidemia en neonatos hospitalizados en la sala de neonatología del Hospital del Niño, enero 2014 a diciembre de 2016. Análisis univariado no pareado

\begin{tabular}{|c|c|c|c|c|}
\hline Variable & $\begin{array}{c}\text { Caso } \\
\mathrm{n}=134\end{array}$ & $\begin{array}{l}\text { Control } \\
n=268\end{array}$ & $\begin{array}{l}\text { Odds Ratio } \\
\text { No pareado }\end{array}$ & Valor $p$ \\
\hline \multicolumn{5}{|l|}{ Estancia Intrahospitalaria } \\
\hline >7 días & 131 & 139 & $40,5(12,9-202,6)$ & 0,00 \\
\hline UCIN & 130 & 230 & $5,3(1,8-21,1)$ & 0,00 \\
\hline Ventilación Mecánica & 128 & 202 & $6,9(2,9-20,1)$ & 0,00 \\
\hline \multicolumn{5}{|l|}{ Medicamentos } \\
\hline \multicolumn{5}{|l|}{ Antibiótico amplio espectro } \\
\hline Piperacilina/Tazobactam & 118 & 85 & $15,8(8,6-30,2)$ & 0,00 \\
\hline Meropenem & 107 & 42 & $21,3(12,0-37,8)$ & 0,00 \\
\hline \multicolumn{5}{|l|}{ NPT } \\
\hline$>5$ días & 132 & 185 & $29,6(7,6-251,6)$ & 0,00 \\
\hline \multicolumn{5}{|l|}{ Procedimientos } \\
\hline \multicolumn{5}{|l|}{ Invasivos } \\
\hline Líneas umbilicales & 101 & 155 & $2,2(1,3-3,6)$ & 0,00 \\
\hline Percutáneo & 84 & 32 & $12,3(7,2-21,3)$ & 0,00 \\
\hline Catéter Venosos & 21 & 7 & $6,9(2,7-19,7)$ & 0,00 \\
\hline \multicolumn{5}{|l|}{ Central } \\
\hline \multicolumn{5}{|l|}{ Cirugía } \\
\hline Abdominal & 37 & 10 & $9,8(4,5-22,9)$ & 0,00 \\
\hline Torácica & 9 & 5 & $3,7(1,1-14,6)$ & 0,01 \\
\hline Enterocolitis & 25 & 19 & $3,0(1,5-6,0)$ & 0,00 \\
\hline \multicolumn{5}{|l|}{ Necrotizante } \\
\hline Crecimiento bacteriano hemocultivo previo candidemia & 34 & 35 & $2,2(1,2-3,9)$ & 0,00 \\
\hline
\end{tabular}

UCIN: Unidad de Cuidados Intensivos Neonatal; NPT: Nutrición Parenteral. 
que se mantienen las mismas variables como factores de riesgo, estadísticamente significativas para candidemia, identificando la estancia intrahospitalaria mayor a 7 días, el uso de antibióticos de amplio espectro, ingreso a UCIN y la exposición a ventilación mecánica, el uso de nutrición parenteral por más de 5 días, procedimientos invasivos como colocación de accesos venosos centrales y cirugías abdominales y torácicas, enterocolitis necrosante y crecimiento de microorganismo bacteriano en hemocultivo previo a la candidemia. De los factores de riesgo estadísticamente significativos, los que representan mayor riesgo son la estancia intrahospitalaria mayor a 7 días, el uso de nutrición parenteral por más de 5 días y la exposición a cirugías abdominales (tabla 4).

\section{Análisis multivariado}

En el análisis multivariado se identificaron como factores de riesgo la estancia intrahospitalaria mayor a 7 días, el uso de líneas umbilicales, la exposición a cirugía y el manejo con meropenem (tabla 5).

Tabla 4. Factores de Riesgo para Candidemia en neonatos hospitalizados en la sala de neonatología del Hospital del Niño, enero de 2014 a diciembre de 2016. Análisis univariado pareado

\begin{tabular}{|c|c|c|c|}
\hline Variable & Odds Ratio Pareado & Intervalo de Confianza 95\% & Valor $p$ \\
\hline \multicolumn{4}{|l|}{ Estancia Intrahospitalaria } \\
\hline$>7$ días & 54,9 & $13,4-224,7$ & 0,00 \\
\hline UCIN & 6,0 & $2,0-17,5$ & 0,00 \\
\hline Ventilación Mecánica & 9,1 & $3,5-23,5$ & 0,00 \\
\hline \multicolumn{4}{|l|}{ Medicamentos } \\
\hline \multicolumn{4}{|l|}{ Antibiótico amplio espectro } \\
\hline Piperacilina/Tazobactam & 3,1 & $2,3-3,9$ & 0,00 \\
\hline Meropenem & 2,8 & $2,1-3,5$ & 0,00 \\
\hline \multicolumn{4}{|l|}{ NPT } \\
\hline$>5$ días & 30,2 & $7,3-125,1$ & 0,00 \\
\hline \multicolumn{4}{|l|}{ Procedimientos Invasivos } \\
\hline Líneas umbilicales & 2,8 & $1,6-4,9$ & 0,00 \\
\hline Percutáneo & 10,9 & $6,0-19,7$ & 0,00 \\
\hline Catéter venosos central & 6,7 & $2,7-16,8$ & 0,00 \\
\hline \multicolumn{4}{|l|}{ Cirugía } \\
\hline Abdominal & 16,5 & $5,8-46,6$ & 0,00 \\
\hline Torácica & 5,0 & $1,3-19,1$ & 0,01 \\
\hline Enterocolitis Necrotizante & 2,9 & $1,5-5,4$ & 0,00 \\
\hline Crecimiento Bacteriano hemocultivo previo candidemia & 2,0 & $1,2-3,4$ & 0,00 \\
\hline
\end{tabular}

UCIN: Unidad de cuidados intensivos neonatal; NPT: Nutrición parenteral.

Tabla 5. Factores de Riesgo para Candidemia en neonatos hospitalizados en la sala de neonatología del Hospital del Niño, enero de 2014 a diciembre de 2016. Análisis multivariado pareado

\begin{tabular}{|c|c|c|c|c|}
\hline \multicolumn{2}{|l|}{ Variable } & \multirow{2}{*}{$\frac{\text { Odds Ratio (OR) }}{14,2}$} & \multirow{2}{*}{$\frac{\text { Intervalo Confianza 95\% }}{1,5-128,4}$} & \multirow{2}{*}{$\frac{\text { Valor } p}{0,01}$} \\
\hline Edad gestacional & $<32$ semanas & & & \\
\hline Peso al nacer & $<1.000 \mathrm{~g}$ & 8,5 & $1,6-44,3$ & 0,01 \\
\hline \multicolumn{2}{|c|}{ Estancia intrahospitalaria } & 17,0 & $2,3-122,4$ & 0,00 \\
\hline \multicolumn{2}{|l|}{ UCIN } & 0,4 & $0,0-2,8$ & 0,36 \\
\hline \multicolumn{2}{|l|}{ Percutáneo } & 1,2 & $0,3-3,9$ & 0,69 \\
\hline \multicolumn{2}{|l|}{ NPT } & 6,1 & $0,5-68,9$ & 0,13 \\
\hline \multicolumn{2}{|l|}{ Líneas umbilicales } & 9,0 & $1,5-52,5$ & 0,01 \\
\hline \multicolumn{2}{|c|}{ Catéter venosos central } & 1,4 & $0,2-8,8$ & 0,67 \\
\hline \multicolumn{2}{|l|}{ Cirugía } & 12,4 & $1,7-87,3$ & 0,01 \\
\hline \multicolumn{5}{|c|}{ Antibiótico amplio espectro } \\
\hline \multicolumn{2}{|l|}{ Meropenem } & 4,6 & $1,3-15,9$ & 0,01 \\
\hline \multicolumn{2}{|c|}{ Piperacilina Tazobactam } & 3,7 & $0,9-14,8$ & 0,05 \\
\hline
\end{tabular}

UCIN: Unidad de Cuidados Intensivos Neonatal; NPT: Nutrición Parenteral. 


\section{Discusión}

El estudio logró evaluar los factores de riesgo más importantes asociados a candidemia en los pacientes hospitalizados en la sala de neonatología: estancia intrahospitalaria mayor a 7 días, uso de líneas umbilicales, exposición a cirugía y tratamiento con meropenem. A pesar de que existen limitaciones para evaluar los factores de riesgo, se decidió realizar un estudio de casos y controles pareado para disminuir posibles sesgos.

La tasa de incidencia hospitalaria de Candida en el estudio fue de 20 por cada 1.000 admisiones, siendo elevada al compararse con otros estudios como el de Feja et al. ${ }^{6}$ en Nueva York, Fu et al. ${ }^{9}$ y Chen et al. ${ }^{10}$ en China y Pinhat et al. ${ }^{11}$ en Brasil siendo 15.9, 13.6, 10.6 y 9.0 por cada 1.000 admisiones respectivamente. Dentro de las posibles razones asociadas a la elevada incidencia de candidemia en nuestra institución, se pueden mencionar: el hacinamiento diario (censo por arriba de la capacidad), el poco personal médico y de enfermería (relación numérica por paciente), la alta complejidad de las patologías, ser un centro de referencia nacional y el uso indiscriminado de antibióticos de amplio espectro. De acuerdo a los resultados obtenidos, recomendamos disminuir el uso de antibióticos de amplio espectro, especialmente los carbapenémicos, mejorar las técnicas asépticas a la hora de colocar y usar las líneas umbilicales e intentar en lo posible disminuir los días intrahospitalarios para así reducir la incidencia de candidemia en la institución.

El 49\% de los casos de candidemia fue causado por Candida parapsilosis similar a lo expuesto en los estudios Yu et al. ${ }^{12}$, Rodríguez et al. ${ }^{13}$, Neu et al..$^{14}$, Celebi et al. ${ }^{15} \mathrm{y}$ Caggiano et al. ${ }^{16} \mathrm{~A}$ diferencia, otros estudios, Feja et al. ${ }^{6}$, J. Fu et al. ${ }^{9}$, Chen et al. ${ }^{10}$, Saiman et al. ${ }^{17}$, Robinson et al. ${ }^{18}$, Juyal et al. ${ }^{19}$, Hsu et al. ${ }^{20}$ y Márquez et al. ${ }^{21}$, donde se describe que la especie de predominio fue Candida albicans.

La tasa de mortalidad global en la sala de neonatología de nuestra institución, en pacientes menores de $2.500 \mathrm{~g}$, es de $10 \%$ (fuente: base de datos de neonatología). Durante el periodo de estudio, la tasa de letalidad global por candidemia fue de $62 \%$. Candida parapsilosis fue responsable del mayor número de episodios y de letalidad por candidemia.

Los factores de virulencia de la Candida parapsilosis incluyen características bioquímicas y procesos metabólicos como la producción de fosfolipasas, la síntesis y almacenamiento de ácidos grasos, gran afinidad por superficies sintéticas (mayor que Candida albicans) y diferencias en la estructura y composición de las biopelículas, que contribuyen al aumento del número de casos observado en los últimos años. ${ }^{22-24}$ Además la menor susceptibilidad a diversos antifúngicos como anfotericina $\mathrm{B}$, fluconazol y caspofungina, junto con la aparición de cepas multirresistentes contribuye a la mayor incidencia de dicha especie comparada con las otras especies de Candida ${ }^{25}$.

En el estudio no se encontró relación entre la persistencia de candidemia y la mortalidad, similar a lo obtenido por Robinson et al. ${ }^{18}$, Levy et al. ${ }^{26}$ y Benjamin et $\mathrm{al}^{21}$.

En el análisis multivariado se identificaron como factores de riesgo: la estancia intrahospitalaria, el uso de líneas umbilicales, la exposición a cirugía y el manejo con meropenem. Estos factores de riesgo aparecen como estadísticamente significativos en la literatura; la intervención quirúrgica como factor de riesgo ha sido mencionada previamente en el estudio de ÁvilaAgüero et al. ${ }^{28}$ en base a la disrupción de las barreras cutáneas y epiteliales para la invasión del torrente sanguíneo por Candida. La estancia intrahospitalaria y el uso de meropenem han sido identificados como factores de riesgo en estudios por Fu et al. ${ }^{9}$, Chen et al. ${ }^{10}$, $\mathrm{Yu}$, et al. ${ }^{12}$, Saiman et al. ${ }^{17}$, Stoll et al. ${ }^{29}$, Benjamin et al. ${ }^{30}$ y Hsu et al. ${ }^{31}$ Se ha encontrado que los antibióticos carbapenémicos (Meropenem) pueden contribuir a la proliferación de microorganismos oportunistas como Candida, al reducir la presión competitiva ejercida por la flora bacteriana normal. ${ }^{9}$ Un estudio por Kaufman et al. ${ }^{32}$ mostró que la disminución del uso de carbapenémicos está asociado al descenso de la incidencia de infecciones por Candida.

En el estudio el principal factor de riesgo fue la estancia intrahospitalaria ya que provee la oportunidad de Candida a colonizar a los pacientes. Estos resultados coinciden con los encontrados en otras unidades neonatales 33,34 .

Además se identificó la colocación de líneas umbilicales como un factor de riesgo para el desarrollo de candidemia, ya que facilita la introducción de Candida y la fijación a un material extraño por ende formando la biopelículas que conducen a su transmisiónn horizontal ${ }^{35-37}$.

Llama la atención la alta incidencia de candidemia observada en los pacientes con peso menor a $1.250 \mathrm{~g} \mathrm{a}$ pesar de la profilaxis con fluconazol. Entre las posibles explicaciones de este hallazgo hay que considerar un alto grado de transmisión horizontal por inclumplimiento de las normas de bioseguridad, hacinamiento en la sala de neonatología y complejidad de la población neonatal en la región. Se requieren estudios a futuro para evaluar estas posibilidades.

Dentro de las fortalezas del estudio se puede mencionar una adecuada selección y definición de los casos y controles, buen acceso a los expedientes y control de sesgos durante el análisis estadístico.

La importancia de identificar los factores que incrementan las probabilidades de desarrollar candidemia 
se resalta al considerar el impacto que las mismas tienen sobre la morbilidad y mortalidad en la población neonatal. Es imperativo por lo tanto realizar un diagnóstico oportuno e iniciar un tratamiento temprano.

\section{Responsabilidades éticas}

Protección de personas y animales: Los autores declaran que los procedimientos seguidos se conformaron a las normas éticas del comité de experimentación humana responsable y de acuerdo con la Asociación Médica Mundial y la Declaración de Helsinki.

Confidencialidad de los datos: Los autores declaran que han seguido los protocolos de su centro de trabajo sobre la publicación de datos de pacientes.

Derecho a la privacidad y consentimiento informado: Los autores han obtenido el consentimiento in- formado de los pacientes y/o sujetos referidos en el artículo. Este documento obra en poder del autor de correspondencia.

\section{Conflicto de intereses}

Los autores declaran no tener conflicto de intereses. Rodrigo DeAntonio es empleado de GSK Vacunas. Las opiniones expresadas en este artículo son propias y no reflejan las de GSK Vacunas.

\section{Agradecimientos}

Lic. Julio Nieto Ramos por apoyar con las estadísticas de candidemia reportadas en la sala de neonatología, desde enero de 2014 a diciembre de 2016 y al Dr. Joel Méndez por el apoyo en el análisis de los resultados del estudio.

\section{Referencias}

1. Waggoner-Fountain L, Walker M, Hollis R, et al. Vertical and horizontal transmission of unique Candida species to premature newborns. Clin Infect Dis 1996;22(5):803-8.

2. Bendel C. Colonization and epithelial adhesion in the pathogenesis of neonatal candidiasis. Semin Perinatol. 2003;27(5):357-64.

3. Chapman R, Faix R. Persistently positive cultures and outcome in invasive neonatal candidiasis. Pediatr Infect Dis J. 2000;19(9):822-7.

4. Benjamin D Jr, Poole C, Steinbach W, et al. Neonatal candidemia and endorgan damage: a critical appraisal of the literature using meta-analytic techniques. Pediatrics. 2003;112(3 Pt1):634-40.

5. Hammoud M, Al-Tajar A, Fouad M, et al. Persistent candidemia in neonatal care units: risk factors and clinical significance. Int J Infect Dis. 2013;17(8):624-8.

6. Feja K, Wu F, Roberts K, et al. Risk factors for candidemia in critically ill infants: a matched case-control study. J Pediatr. 2005;147(2):156-61.

7. Rueda K, Moreno M, Espinosa M, et al. Impact of routine fluconazole prophylaxis for premature infants with birth weights of less than 1250 grams in a developing country. Pediatr Infect Dis J. 2010;29(11):1050-2.

8. International Ethical Guidelines for Epidemiological Studies - CIOMS. Geneva, February 2008.

9. Fu J, Wang X, Wei B, et al. Risk factors and clinical analysis of candidemia in very-low-birth-weight neonates. Am J Infect Control. 2016;44(11):1321-5.

10. Chen J, Jiang Y, Wei B, et al. Epidemiology of and risk factors for neonatal candidemia at a tertiary care hospital in western China. BMC Infect Dis. 2016;16:700.

11. Pinhat E, Borba M, Ferreira M, et al. Fungal colonization in newborn babies of very low birth weight: a cohort study. J Pediatr (Rio J). 2012;88(3):211-6.

12. Yu Y, Du L, Yuan T, et al. Risk factors and clinical analysis for invasive fungal infection in neonatal intensive care unit patients. Am J Perinatol. 2013;30(07):58994.

13. Rodríguez D, Almirante B, Park B, et al. Candidemia in neonatal intensive care units: Barcelona, Spain. Pediatr Infect Dis J. 2006;25(3):224-9.

14. Neu N, Malik M, Lunding A, et al. Epidemiology of candidemia at a children's hospital, 2002 to 2006. Pediatr Infect Dis J. 2009;28(9):806-9.

15. Celebi S, Hacimustafaoglu M, Koksal N, et al. Neonatal candidiasis: Results of an 8 year study. Pediatr Int. 2012;54(3):341-9.

16. Caggiano G, Lovero G, De Giglio O, et al. Candidemia in the Neonatal Intensive Care Unit: A Retrospective, Observational Survey and Analysis of Literature Data. Biomed Res Int. 2017;2017:7901763.

17. Saiman L, Ludington E, Dawson J, et al. Risk factors for Candida species colonization of neonatal intensive care unit patients. Pediatr Infect Dis J. 2001;20(12):1119-24.

18. Robinson J, Pham H, Bloom B, et al. Risk factors for persistent candidemia infection in a neonatal intensive care unit and its effect on mortality and length of hospitalization. J Perinatolol. 2012;32(8)621-5.

19. Juyal D, Adekhandi S, Negi V, et al. An outbreak of neonatal candidemia due to non-albicans Candida species in a resource constrained setting of Uttarakhand State, India. J Clin Neonatol. 2013;2(4):183-6.

20. Hsu J, Lai M, Lee C, et al. Comparison of the incidence, clinical features and outcomes of invasive candidiasis in children and neonates. BMC Infectious Diseases. 2018;18:194.

21. Márquez F, Iturrieta I, Calvo M, et al. Epidemiología y susceptibilidad antifúngica de especies causantes de candidemia en las ciudad de Valdivia, Chile. Rev Chil infectol. 2017;34(5): 441-6.

22. Chow B, Linden J, Bliss J. Candida parapsilosis and the neonate: epidemiology, virulence and host defense in a unique patient setting. Expert Rev Anti Infect Ther 2012;10(8):935-46.

23. Toth R, Toth A, Vagvolgyi C, et al. Candida parapsilosis Secreted Lipase as an Important Virulence Factor. Curr Protein Pept Sci. 2017;18(10):1043-9.

24. Pharkjaksu S, Chongtrakool P, Suwannakarn K, et al. Species distribution, virulence factors, and antifungal susceptibility among Candida parapsilosis complex isolates from clinical specimens at Siriraj Hospital, Thailand, from 2011 to 2015. Med Mycol. 2018;56(4):426-33.

25. Prigent G, Ait-Ammar N, Levesque 
E, et al. Echinocandin Resistance in Candida Species Isolates from Liver Transplant Recipients. Antimicrob Agents Chemother. 2017;61(2):e01229-16.

26. Levy I, Shalit I, Askenazi S, et al. Duration and outcome of persistent candidemia in newborn infants. Mycoses. 2006;49(3):197-201.

27. Benjamin D, Hudak M, Duara S, et al. Effect of fluconazole prophylaxis on candidiasis and mortality in premature infants: a randomized clinical trial. JAMA. 2014;311(17):1742-9.

28. Ávila-Agüero M, Canas-Coto A, Ulloa Gutiérrez R, et al. Risk factors for Candida infections in a neonatal intensive care unit in Costa Rica. Int J Infect Dis. 2005;9(2):90-5.

29. Stoll B, Hansen N, Fanaroff A, et al.
Late-onset sepsis in very low birth weight neonates: the experience of the NICHD Neonatal Research Network. J Pediatr. 2002;110(2):285-91.

30. Benjamin D Jr, DeLong E, Steinbach W, et al. Empirical therapy for neonatal candidemia in very low birth weight infants. J Pediatr. 2003;112(3):543-7.

31. Hsu J, Lai M, Lee C, et al. Comparison of the incidence, clinical features and outcomes of invasive candidiasis in children and neonates. BMC Infectious Diseases. 2018;18:194.

32. Kaufman D. Challenging issues in neonatal candidiasis. Curr Med Res Opin. 2010; 26(7):1769-78.

33. Basu S, Kumar R, Tilak R, et al. Candida Blood Stream Infection in Neonates: Experience from A Tertiary Care Teaching
Hospital of Central India. Indian Pediatr. 2017;54(7):556-9.

34. James M, Anuja J, Ninan P. Clinical profile of neonatal candidiasis in newborn nursery. Int J Contemp Pediatr. 2018;5(2):334-7.

35. Bouza E, Guinea J, Guembe M. The Role of Antifungals against Candida biofilm in catheter-related candidemia. Antibiotics (Basel). 2015;4(1):1-17.

36. Lee J. Catheter-related bloodstream infections in neonatal intensive care units. Korean J Pediatr. 2011;54(9):363-7.

37. Pammi M, Zhong D, Johnson Y, et al. Polymicrobial bloodstream infections in the neonatal intensive care unit are associated with increased mortality: a case-control study. BMC Infect Dis. 2014;14:390. 